\title{
Research of Electric Welding Machine's Tractor System Based on Digital Signal Processor
}

\author{
Li-li Ding ${ }^{1}$, Jin $\mathrm{Hu}^{2}$ \\ The First Aeronautic Institute of Air Force, Xinyang 464000, China \\ 1email: lilac6212@sina.com, ${ }^{2}$ email:benchi9506@sina.com
}

Keywords: Electric Welding Machine; DSP; Brushless DC Motor; Tractor System

\begin{abstract}
It expatiates on the design of TMS320F240DSP in wire feeding system which introduces the thoughts of design, hardware and software to the system. And it also presents the application of brushless DC motor in wire feeding system.
\end{abstract}

\section{Introduction}

Welding trolleys and wire feeding mechanism are essential to drag the system in various types of automatic and semi-automatic welding equipments which have a direct impact on their stability and reliability of the welding quality. At present, there are many circuits of welding speed and wire feed drive circuit which are commonly used, such as transistor circuits and PWM (Width - Modulation Pulse) circuit, etc. There drag system simulators are easy to aging, and are sensitive to temperature changes, so the maintenance is inconvenient. Although it has partially solved the above problems using MCU control of the drive system, it is difficult to achieve requirements of real-time control for speed by its single-chip processing capability and speed limit. Some power of the PI controllers and PWM still use analog circuits, and digital characteristics have not been fully reflected.

DSP (digital signal processor) chip has a broad application prospect in digital welding field by the stability of the digital devices and large-scale integrated, especially by its easily to be programmable and to realize real-time signal processing. In recent years, it is integrated into a whole in the permanent magnet brushless DC (BLDC) motor with using DSP chip driver and controller, which controlling system is relative simpleness, low cost, high efficiency, energy saving and stable speed through software programming realizes precise speed regulation. It is studied the design and application of DSP and BLDC in the wire drive system of electric welding machine, and the results show that the system can obviously improve the static performance of the system.

\section{Overall Design Ideas}

The wire feeding system of arc welding machine adopts DSP as controller and brushless DC motor as the driving motor, which not only has the ability to extract and process signals in real time, but also integrates the motor control peripheral components. The system is mainly composed of DSP, brushless DC motor, three-phase inverter, position detection and current detection. The schematic diagram of the system is shown in Figure 1. Using DSP controlling, it will record some of the best parameter matching values and will combine these discrete points with different welding conditions, such as wire type, diameter and welding material, etc. At the same time, these welding parameters would be saved FLASH of DSP memory. In the actual welding process, the operator may conveniently operate the machine to select wire feeding speed through the control panel to set the wire types and diameters, arc voltage and arc current, arc force parameters. In this way, the control system has the advantages of simple structure, high reliability and expansibility. In order to drive brushless DC motor constantly turning, it adopts velocity negative feedback. The system adopts PWM mode to realize the brushless motor control which can be eliminated the influence of the welding to send wire resistance and be advantage to feeding wire arc slowly, improving the ignited arc success rate. The basic principle is that the output PWM signals transfer to the power drive circuit for adjusting speed of brushless electric machine through the three-phase inverter. It has the 
function of fault monitoring and self- diagnosis. Once the fault occurs, the PDPINT port is interrupted by the fault protection circuits, and the PWM output is blocked until the fault is eliminated. The controller in the forward path of the control system adopts the mature PID control algorithm, and the DSP is used to realize the digital control, which makes the system more flexible, and the advantages are more easy to play. The control system diagram is shown in Figure 2. It consists of three control loops: position loop, speed loop and current loop. Position loop obtains the rotor position to carry out the correct current. When the ring of current regulates it could adjust the output duty cycle of PWM chopped wave according to the difference between the measured current and the calculated value, then output the correct trigger pulse pair on the basis of the motor starting current and the maximum current for amplitude limiting. The speed loop is adjusted in the light of the velocity feedback and the given value, the system has good steady-state performance.

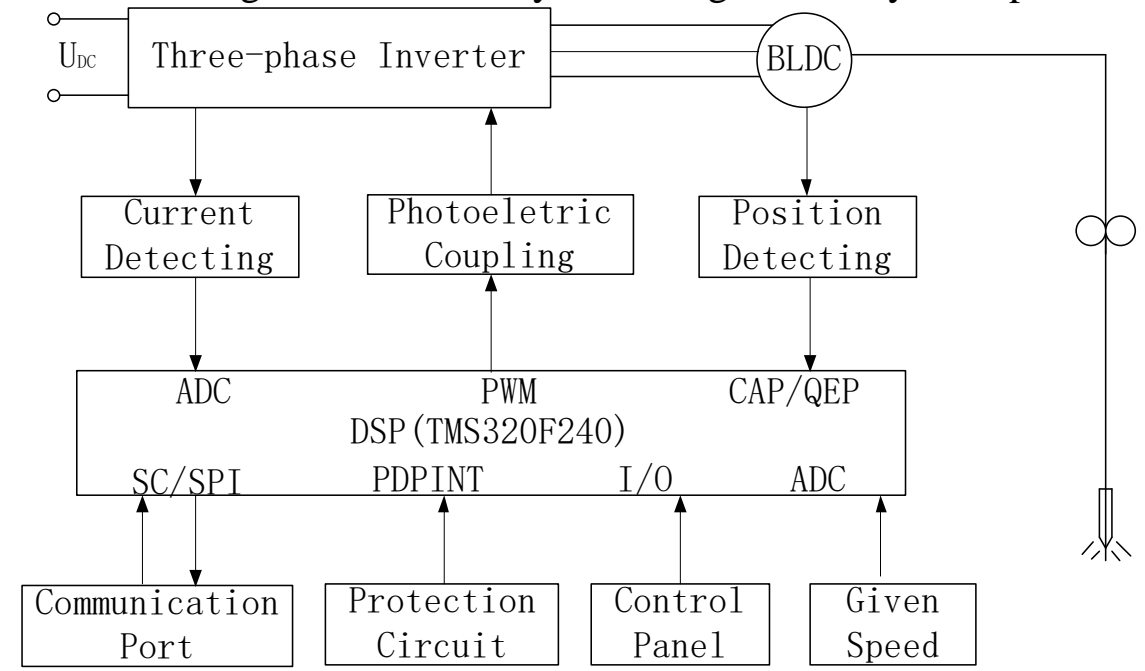

Fig.1 Principle diagram of constant speed wire feeding system based on DSP



Fig.2 Diagram of control system

\section{Hardware Design Of Control System}

Brief Introduction Of DSP. The system uses the chip ---TMS320F240DSP which is designed for motor control by American TI company. It uses Harvard structure which is provided with independent of the read /write data bus and address bus. It also adopts parallel program and data operand addressing support, which many complex operations could be made by lower power consumption, higher reliability and quiet operation. This highly integrated chip quickly becomes a cheap alternative to the traditional micro control unit (MCUS) and expensive multi chip design, the price lowers from several hundred dollars to a few dollars. It has the characteristics of powerful digital processor. For instance, the TMS320F240 DSP controller with the speed of 20 MIPS (millions of instructions per second) can be $8 \sim 10$ times faster than a 16-bit micro controller to deal with the single instruction execution time, completing a multiply add operation $16 \sim 30$ times faster than before. It has a 32-bit accumulator, $16 \mathrm{k} \times 16$ FLASH ROM, 544×16 RAM, three timers, external memory interface modules (EMIF), a 16-bit address bus and a16-bit data bus. It has the unique resources of motor control chip solutions with 12-way pulse width modulation (PWM) output, 2-way A/D with 10-bit of 8 channels, programmable dead zone control unit, four capture units(Captures/QEP), two of which are pulse function interfaces with quadrature encoder. Of course, it also has 28 independent programming multiplexed I/O pins, serial communication interfaces (SCI) 
and serial external equipment interface (SPI) units.

Brushless DC Motor (BLDC).BLDC is a novel electromechanical integrated motor in recent years with the development of power electronic devices and permanent magnetic materials using electronic commutation instead of brush commutation. It not only has the AC motor's characteristics with simple structure, reliable operation and convenient maintenance, but also overcomes the inherent weaknesses with DC servo motor in the electric welding machine to send wire drive system, solving the relative sliding and wearing between brushes and commutators which it is easy to cause some faults, such as loose connection and spark failure. It has been widely used and there is a trend to replace the brush motor because of BLDC with some advantages such as based on its small size, light weight, less calorific value and almost not affected by the fluctuation of power network voltage. In essence, BLDC is composed of three main parts: a controller, a rotor position detection and the motor body. The working principle of BLDC is to change trigger combined state of power device which is controlled by the different position of the rotor to control winding performing a turn-on operation, so as to achieve the automatic control synchronization operation. Similar to a DC motor, the critical factor to the effective torque and velocity control is the relationship between back electromotive force and torque which can indicate respectively.

$$
\begin{aligned}
& E=2 N \operatorname{lr} B \omega \\
& T=\frac{1}{2} i^{2} \frac{d L}{d \theta}-\frac{1}{2} B^{2} \frac{d R}{d \theta}+\frac{4 N}{\pi} B r l \pi i
\end{aligned}
$$

In the above formula, $\mathrm{N}$ is said to self-inductance coefficient; 1 indicates the rotor position; $\mathrm{r}$ is represented as inner diameter of rotor; B is expressed as magnetic flux density of rotor; $\omega$ is Motor angular velocity; $\mathrm{L}$ is self-inductance coefficient; $\theta$ is rotor position; and $\mathrm{R}$ is phase resistance.

Two terms of the torque expression are parasitic reluctance torque factors, and the third term can produce an effective torque. So the motor speed is proportional to the back EMF and torque is almost directly proportional to phase current. Based on this, we may obtain the closed-loop control block diagram, as it is shown in Figure 2.

Three-Phase Inverter. It is realized by the three-phase full controlled bridge inverter to keep the rotor and stator magnetic field synchronous as well as the control of the square wave current of the stator for BLCD. Three phase inverter- motor structure diagram is shown in Figure 3. Motor stator winding adopts star connection, and the space position is symmetrical. Six transistors (IGBT1 -IGBT6) conduction is controlled by PWM unit of DSP, and to determine whether to be conduction so as to control each phase of the motor stator winding for the order and time of electric motor. So that the motor can run stably.

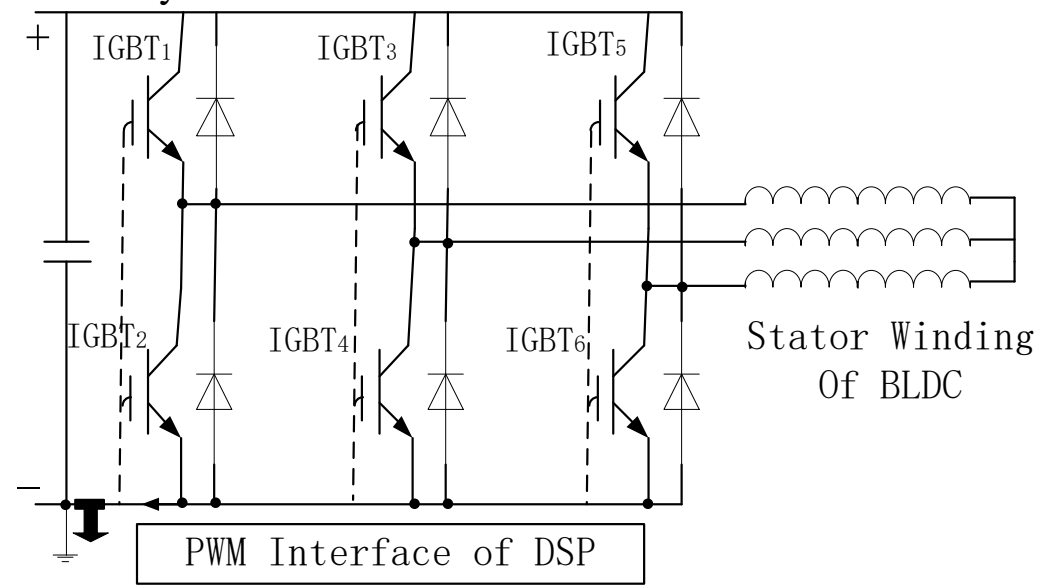

Fig.3 Structure diagram of three-phase inverter and motor

Current Detection. Sampling resistor is used for current detection which is located in the three-phase inverter between low voltage terminal and ground (as is shown in Figure 3). The resistance is determined by the maximum current to the motor. That is, the maximum pressure drop on the resistance is not more than $0.5 \mathrm{~V}$. The voltage on the sampling resistor is changed to the digital signal of the current through the ADC unit on the DSP. In order to achieve $20 \mathrm{kHz}$ of the 
current loop, each of the $50 \mathrm{~s}$ current must be sampled once according to the current measurement values in the PWM cycle to determine the new PWM duty cycle, which generates a new PWM waveform to control the brushless motor.

Speed Sampling And Adjustment. Using three Hall effect position sensor to detect the rotor position and speed of the motor is calculated, as it is shown in Figure 1. The output of the position sensor is directly connected to TMS320F240 capture unit, and the Hall sensor outputs three signals overlapping $180^{\circ}$. The signal can get 6 phase alternating time, so every two signals alternating phase difference is $60^{\circ}$. And because brushless DC motor speed expression can be written as $\Delta \theta /$

$\Delta T$, and Hall sensor relative to the position of a motor is fixed, that is t say, two of the phase difference between the alternating signals $\Delta \theta$ is $60^{\circ}$. So the speed calculation can be simplified into a simple division. The speed can be calculated by measuring the time interval between the two alternating signals. The frequency can be modified according to the use of the speed value when the speed loop is executed once every $2-5$ second. The reference velocity is directly written into the storage space and speed feedback signal with a given reference velocity subtraction obtained velocity error. A new reference current can be obtained by simple PI algorithm.

\section{Software Design}

Software Structure.The whole control system is composed of the initialization procedure and the running interrupt program. The initialization procedure mainly completes the event management system initialization and variable initialization only when the motor starts. The running interrupt program includes CAP/QEP interrupt, ADC conversion end interrupt and PDPINT interrupt. The CAP/ QEP interrupt is mainly used to get the current position of the rotor and the motor speed and adjust the PWM output phase sequence among them. However, the PDPINT interrupt occurs when a fault occurs, stopping the PWM generation and output and setting the error flags to ensure the security of the system. ADC interrupt took place in each PWM cycle occurs after a period of time, through the ADC values calculated after getting the phase current value. After current loop regulation, once the current loop is adjusted, a speed loop is adjusted to ensure system running according to the requirement. ADC interrupt program flow chart is as shown in Figure 4.

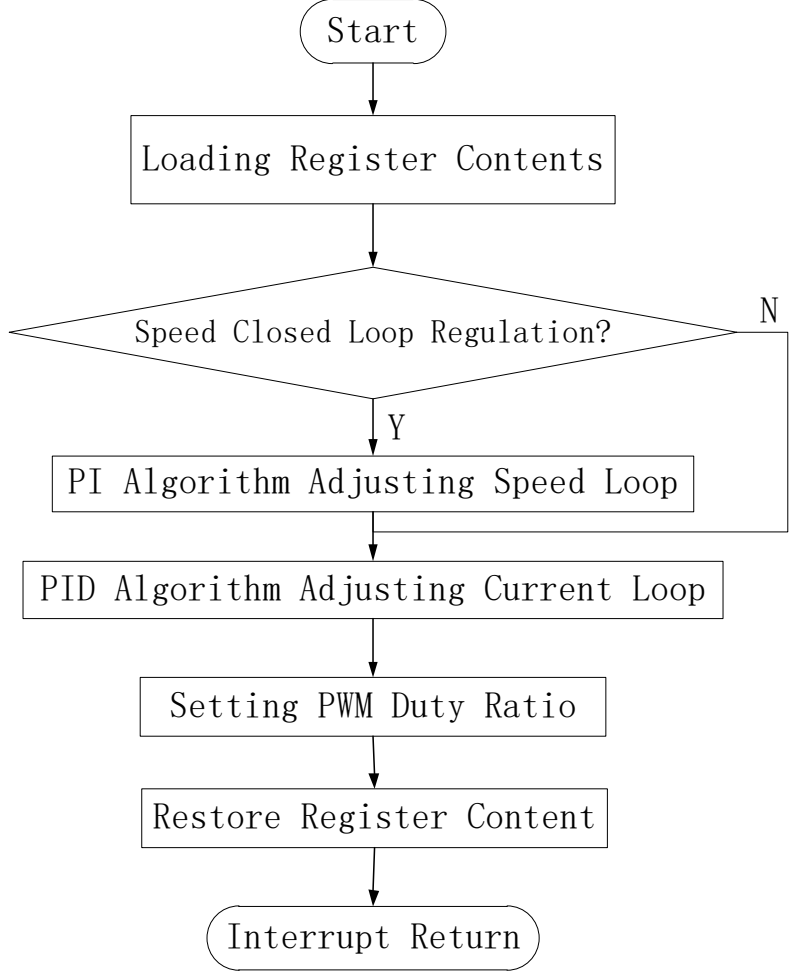

Fig.4 ADC interrupt program flow chart 
Software Programming.Requirements of wire automatic adjustment of the key program is the subroutine speed adjustment. The speed sampling frequency may be determined according to the actual situation. We select $0.03125 \mathrm{~s}$ here, that is, the integral coefficient $\mathrm{Ki}$ is shifted to the right 5 bits which can be obtained. All the variables of the speed loops are stored in a stack. Usually with the auxiliary register AR2in DSP as the stack pointer the first address of the stack can be defined according to the needs of their own. Because the control software and the adjusting parameter are written in FLASH, the modification of the software and the optimization of the welding parameters become very easy.

\section{Test Result}

Select the BLDC model for J130LWX. The power supply voltage is $36 \mathrm{~V}$, the power is $25 \mathrm{~W}$, and the continuous blocking torque is $3 \mathrm{~N} / \mathrm{m}$. The models of brushless DC motor at $600 \mathrm{r} / \mathrm{min}$ speed running results is shown in Figure 5. The first channel detection is the phase current, the second channel detection is the corresponding phase voltage, the third channel is the value calculated by the software of neutral point voltage, and the fourth channel is a pulse of the software at each zero crossing point given. Test results show that the system can run reliably at low speed (such as $30 \mathrm{r} /$ min), and has a good static performance.

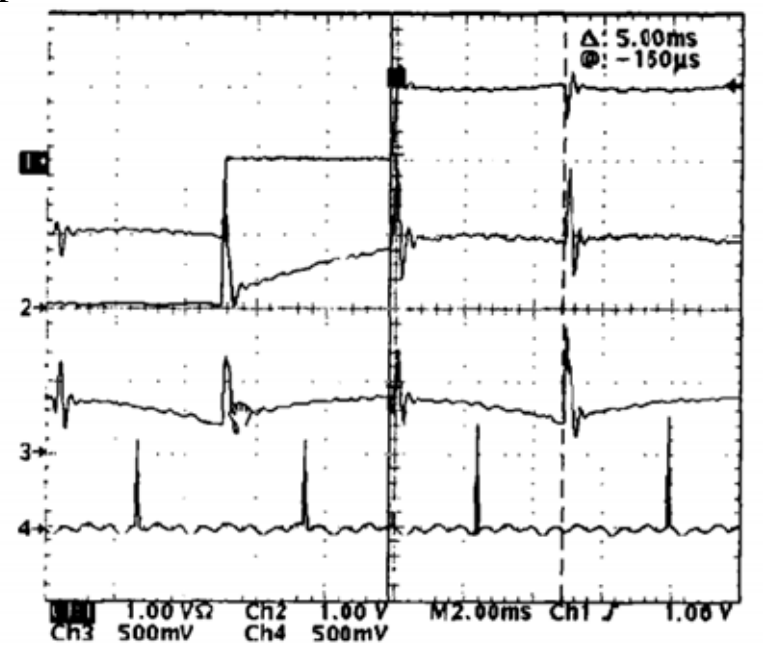

Fig. 5 Operating results of drag system $600 \mathrm{r} / \mathrm{min}$

\section{Conclusion}

It is designed the software and hardware of electric welding machine drive system using the DSP, and the brushless DC motors are used in the drive system of electric welding machine. By the experiment of BLDC motor with the same power DC motor compared temperature that it can be lower by about $30 \%$, and the motor is brushless commutator, which reduces the motor brush wearing, reduces the noise and greatly improves the service life of the motor. If the whole welding machine control system adopts digital control, which can be used PC or microprocessor and DSP combination of two level control, the DSP will complete the regulation and other complex adjustment of the welding power supply and wire feeding speed. The application of BLDC and DSP in the electric welding machine drag system has stable performance, high performance price ratio and broad prospect. It can also be extended to other occasions that need to be adjusted.

\section{References}

[1] Kuan-fang He, Zhi Tan, Yong-Xiao Cheng, ming Dong \& Zhuo-jie Zhang. Key issues of the acoustic emission detection for welding hot crack. Journal of Hunan University of Science \& Technology(Natural Science Edition) ,PP.40-46,2015.

[2] Gao Z G. Numerical modeling to understand liquation cracking propensity during laser and laser 
hybrid welding [J].The International Joumal of Advanced Manufacturing Technology, PP. 291-303, 2012.

[3] Shankar V, Gill T P S, Mannan S L, et al. Criteria for hot cracking evaluation in austenitic stainless steel welds using longitudinal varestraint and transvarestraint tests[J]. Science \& Technology of Weldinig \& Joining,PP.91-97, 2000.

[4] Feng Z Zgaharia T, David S A. Themral stress development in a nikckel based supearlloy during weldability test[J].Welding Jounral, 470-483, 1997.

[5] Shu F Y, Lv Y H, Liu Y X, et al, Residual stress modeling of narrow gap welded joint of aluminum alloy by cold metal transferring procedure [J]. Construction and Building Materials. PP224-235, 2014.

[6] Prikry R, Lokajicek T. Acoustic emission characteristics and failure of unitarily stressed granitic rocks: the effect of rock fabric[J]. Rock Mechanics and Rock Engineering.PP255-270,2003.

[7] G.D.Janaki Ram et al. Effects of pulsed welding current on the solidification structures in Al-Li-Cu and Al-Zn-Mg alloy welds[J]. Practical Metallography,2000,37(5):276-288.

[8] Boldyrev et al. Controlled solidification during fusion welding. Welding production, 1971, 18(6):54-58. 